\title{
State of the Art on Kidney Development: How Nephron Endowment at Birth Can Shape Our Susceptibility to Renal Dysfunction Later in Life
}

\author{
Vassilios Fanos, MD ${ }^{1}$ Clara Gerosa, MD ${ }^{2}$ Cristina Loddo, $\mathrm{MD}^{3}$ Gavino Faa, MD² \\ ${ }^{1}$ Neonatal Intensive Care Unit, AOU, University of Cagliari, \\ Cagliari, Italy \\ 2 Department of Pathology, AOU, University of Cagliari, Cagliari, Italy \\ ${ }^{3}$ Division of Pediatrics, Rovigo Hospital, Rovigo, Italy \\ Address for correspondence Vassilios Fanos, MD, Terapia Intensiva \\ Neonatale, S.S. 554 (bivio Sestu)-Policlinico Monserrato, Blocco \\ Q-09042 Monserrato, Cagliari, Italy (e-mail: vafanos@tin.it).
}

Am J Perinatol 2019;36(suppl S2):S33-S36.

\begin{abstract} Keywords

- nephrogenesis

- kidney development

- chronic kidney disease

- fetal programming of kidney diseases

- renal regenerative medicine

In the present article, we discuss the following topics: (1) the fetal programming of adult kidney diseases and (2) the role of neonatologists in the regenerative renal medicine, based on the activation of resident renal SC. Here, we report the most important steps of our collaboration between neonatologists, nephrologists, and pathologists. Nephrologists should be more interested in clinical data regarding the first month of life in the womb of their adult patients, being particularly focused on birth weight and on the weeks of gestation at birth, without forgetting data regarding maternal status during gestation and neonatal asphyxia. Neonatologists should be aware that any preterm or low birthweight infant should be considered as a subject with fewer glomeruli, probably predicted to develop renal disease later in life.
\end{abstract}

On August 2010, after an international workshop on developmental nephrology, which took place in New Platz (New York), attended by one of the authors (V.F.), a new group of study was defined, characterized by the cooperation among neonatologists, pediatric nephrologists, and pathologists experts in the study of fetal and neonatal human kidney. The aim of this work is to describe 8 years of cooperation, during which the group of pathologists developed a new knowledge on the development of human kidney during gestation, focusing on the characterization of renal stem cells (SCs), whereas pediatric nephrologists were involved in developing new theories on the clinical significance of changes in kidney development, both in the perinatal period and in adulthood. Two main fields of research were involved in our projects: (1) the fetal programming of adult kidney diseases and (2) the role of neonatologists in the regenerative renal medicine, based on the activation of resident renal SC. Here, we report the most important steps of our activity.

\section{The Fetal Programming of Kidney Diseases}

\section{Recent Acquisitions in Kidney Embryology}

Kidney development is a complex process that requires interactions among renal SCs, including metanephric mesenchymal cells and progenitors originating from the ureteric bud (UB) tip. Renal development during gestation generates multiple specialized cell types that characterize the mature kidney architecture. ${ }^{1}$ Nephrogenesis mainly occurs in the subcapsular zone of the fetal kidney, where mesenchymal progenitors addensate, giving rise to a dark area called "blue strip," whose depth may be utilized for a semiquantitative evaluation of the residual nephrogenic potential of antenatal kidney. ${ }^{2}$ Glomerulogenesis and nephrogenesis depend on the interactions between two components emerging from two different developmental structures: the branching tubules originating from the UB and the metanephric mesenchymal cells. ${ }^{1}$ Once arrived in close proximity to the renal capsule, the epithelial mesenchymal cells of the UB tips take in contact with the mesenchymal
Copyright (c) 2019 by Thieme Medical Publishers, Inc., 333 Seventh Avenue, New York, NY 10001, USA. Tel: +1(212) 584-4662.
DOI https://doi.org/ 10.1055/s-0039-1691798. ISSN 0735-1631. 
precursors, which condense giving rise to the cap mesenchymal cells. The induced cap mesenchymal cells of the human kidney have been found to express Bcl-2, an antiapoptotic protein. ${ }^{3}$ The interaction between the cells of the epithelial ampullae and the cap mesenchyme induces the mesenchymal progenitors to undergo mesenchymal-epithelial transition (MET). The morphological sign of this process is represented by the appearance of the renal vesicles, each of which originates a new nephron. ${ }^{1}$ Our studies are at the basis of the theory of the existence of multiple SC niches, each of them might be involved in kidney development, by originating the multiple different renal cell types present in the mature human kidney. ${ }^{4}$

\section{Peculiarities of Nephrogenesis with Clinical Implications}

Two main findings characterize human nephrogenesis, and both might play a relevant role in clinical practice. The first finding is represented by the assumption that nephrogenesis, in physiology, is completed between the 36th and the 38th weeks of gestation. Consequently, the number of nephrons we are born with represents our nephron burden along the whole life. The second significant finding is represented by the ability of infants born before 36 weeks of gestational age (GA), to continue renal maturation and nephrogenesis after birth, but only for a maximum of 2 to 4 weeks. ${ }^{5}$ Therefore, a preterm born at 25 weeks of GA will continue to generate new nephrons until 28 to 29 weeks of GA, losing 9 weeks of nephrogenesis. This infant will not succeed to reach the physiological number of functioning nephrons typical of mature newborns and will become an oligonephronic subject for the whole life. In brief, extreme immaturity at birth is associated with impaired nephrogenesis and with oligonephronia in childhood and in adulthood. ${ }^{6}$

Marked disparities in nephron number should not be restricted to immaturity. Other factors influencing our burden of glomeruli are represented by low birth weight $(\mathrm{LBW})^{7}$ and perinatal nutrient restriction. ${ }^{8}$ A marked interindividual variability in kidney maturation and in nephron number at birth, independent from GA and from body weight at birth, has been reported by our group in preterm and at-term newborns. ${ }^{9}$ According to other authors, the number of glomeruli for individual kidneys might range from approximately 200,000 to 2.5 million, that is, more than 10 times from one individual to the next. $^{10}$

The clinical significance of a pathological event occurring in adult life and destroying part of individual nephron endowment might be completely different, taking into consideration two subjects with a different number of nephrons at birth. All these data taken together, we were forced to face the complex and fascinating theme of fetal programming of the kidney disease occurring in adulthood ${ }^{6,11}$ (-Fig. 1).

\section{Perinatal Regenerative Medicine: A New Challenge for Neonatologists}

\section{A New Frontier: The Blue Strip}

From an analysis of the literature, we realized that the vast majority of studies on the perinatal programming of kidney

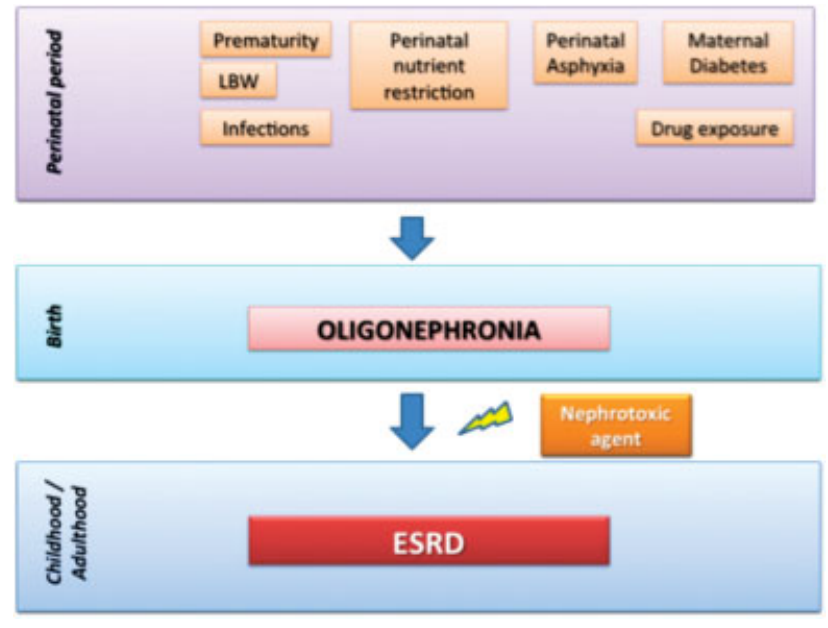

Fig. 1 Perinatal programming of the kidney. Several perinatal factors can influence our number of nephrons at birth. ${ }^{6-8}$ Infants born with fewer functioning glomeruli should be predicted to develop renal disease later in life, when a second "hit," represented by any nephrotoxic agent, would destroy a significant number of nephrons, modifying the ability of the remaining glomeruli to assure a proper renal function. ESRD, end-stage renal disease; LBW, low birth weight.

diseases were focused on the factors involved in intrauterine growth restriction and on the causes of a low nephron number. ${ }^{6}$ Our group decided to focus on the mechanisms that are able to accelerate nephrogenesis in the perinatal period, to reach several nephrons sufficient to counteract kidney failure in adulthood. Given that nephrogenesis in preterm infants may continue for 4 to 6 weeks after birth, our group hypothesized that neonatologists might play a relevant role in the prevention of hypertension and chronic kidney disease (CKD) in adulthood. We defined this new approach as "physiological" renal regenerating medicine, mainly based on the activation of resident renal SCs, prolonging nephrogenesis until 36 to 38 weeks of postconceptual age in all preterm infants. ${ }^{12}$

Our hypothesis underlines a new fundamental function for neonatologists: the necessity of starting a renal regenerative medicine in the perinatal period, particularly in preterm and small-for-date neonates. The reason for this perinatal regenerative approach is based on the histology of the preterm kidney. In fact, the histological analysis of multiple kidneys of infants born prematurely evidenced significant differences from the kidneys of at-term infants. The latter is characterized by a scarcity of stem/progenitor cells, well evidenced by the disappearance of the blue strip. On the contrary, the preterm kidney is characterized by the presence of a huge amount of active endogenous stem/ progenitor cells, well evidenced by a thick blue strip, a condensation of undifferentiated renal stem/progenitors in the subcapsular area.

\section{New Immunohistochemical Data on Renal Progenitors} Studies on the composition of the blue strip, performed by immunohistochemical stains, revealed a previously unreported complexity of this population of renal progenitors, evidencing different steps of differentiation not appreciable 
on simple morphology. ${ }^{3,13}$ These renal progenitor/SCs, so abundant in the immature kidney of preterm infants, represent a unique opportunity for preventing oligonephronia: the knowledge of the multiple markers expressed by mesenchymal SCs might allow neonatologists to induce MET and glomerulogenesis even after birth, changing the fate of renal SC and restoring the nephron burden. ${ }^{14}$

\section{Renal Regenerative Medicine: New Data from Embryology}

The vast majority of studies on kidney regeneration medicine were mainly focused on adult patients affected by CKD with the aim to create a new SC-based approach to treat patients affected with renal failure. ${ }^{11}$ Even the most recent approaches, based on the use of a kidney scaffold and on its recellularization utilizing renal stem/progenitor cells, have encountered many obstacles; the fascinating strategy of renal regenerative medicine is under evaluation by the scientific community. ${ }^{15}$

\section{Epigenetics of Nephrogenesis}

Our proposal starts from an "embryological" view of renal disease: our susceptibility to develop CKD and eventually renal failure in adulthood, starts in utero, and it is mainly due to an abnormal programming of kidney development, ending with a low number of functioning glomeruli. ${ }^{16}$ Moreover, events such as perinatal asphyxia, maternal malnutrition, maternal diabetes, infections, and maternal drug exposure may cause intrauterine growth restriction and sustain reduction of functioning glomeruli with hyperfiltration of the remaining ones, predisposing to individual susceptibility to CKD later in life. ${ }^{17}$

\section{The Two Hits Hypothesis of CKD}

Our hypothesis is that infants born with fewer functioning glomeruli should be predicted to develop renal disease later in life, when a second "hit," represented by any nephrotoxic agent, would destroy a significant number of nephrons, modifying the ability of the remaining glomeruli to assure a proper renal function (-Fig. 1). In brief, it appears plausible that a kidney with an abundant number of glomeruli (e.g., 2 million per kidney) might be able to withstand the multiple injuries that may occur in the lifespan, without significant consequences on the global renal function. At the other extreme of the spectrum, it is plausible that a kidney with 10 times less glomeruli, allowing a sufficient renal function in physiological conditions, will be less able to counteract additional injuries that may occur later in life, ending with renal insufficiency.

\section{Endogenous Stem Cell-Based Renal Regenerative Medicine}

Different from other approaches of regenerative medicine based on the use of renal adult, often exogenous SCs and focused on adult patients, our opinion is that renal regenerative medicine should develop new methodologies, stemmed from the previous attempts. Given that the newborn kidney, particularly in preterm infants, is characterized

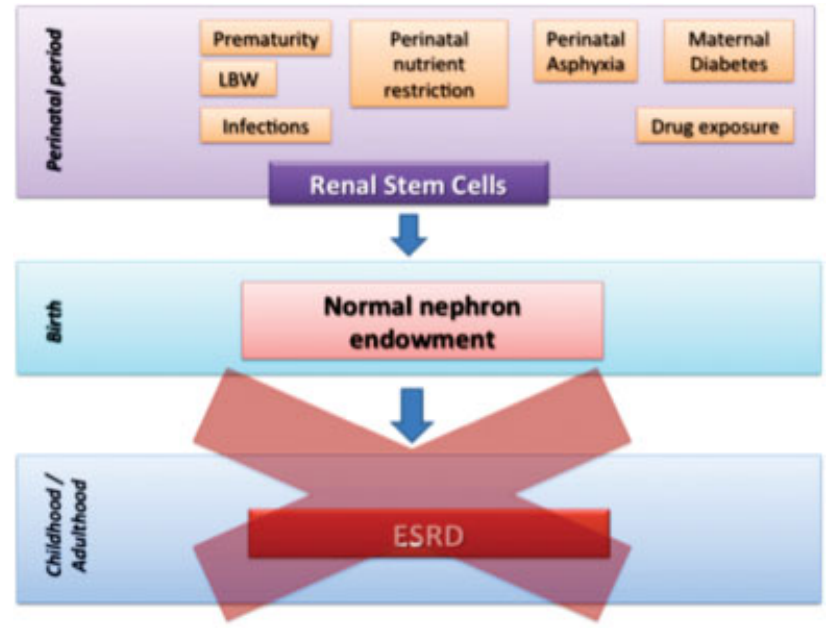

Fig. 2 Physiological renal regenerative medicine. Our proposal is a preventive approach, starting in the perinatal period in preterm and LBW infants, by inducing pluripotent renal SCs to generate new nephrons after birth, thus improving kidney function and protecting them from developing CKD and ESRD later in life. ${ }^{19}$ CKD, chronic kidney disease; ESRD, end-stage renal disease; LBW, low birth weight.; SCs, stem cells.

by a huge number of active stem/precursor cells, the perinatal period represents a fascinating window, for a preventive regenerative approach to CKD. This regenerative proposal contains two main innovative factors, as compared with the classical regenerative approaches. It should be mainly based on endogenous SC, and in particular, on the metanephric mesenchymal cells that are physiologically present, in huge amounts, in the preterm kidney. ${ }^{18}$

\section{Prevention of CKD: Should We Start at Birth?}

A peculiar aspect of our proposal is the preventive approach, starting in the perinatal period in preterm and LBW infants, by inducing pluripotent renal SCs to generate new nephrons after birth, thus improving kidney function and protecting them from developing CKD and end-stage renal disease later in life ${ }^{19}$ (-Fig. 2).

The timing of our regenerative proposal is mandatory, depending on the very limited survival time of renal SCs after birth, probably due to their preference for a hypoxic state that is abruptly interrupted after delivery. This hypothesis of hypoxia as a good factor for renal SCs has been recently supported by an immunohistochemical study from our group, in which we used the antibody CD31 for evidencing vascular structures in fetal kidneys with an active SC component, revealing a scarcity of vessels restricted to the blue strip. $^{20}$

\section{Conclusion}

In conclusion, in our opinion, times are changing both for nephrologists and neonatologists, regarding regenerative medicine and prevention of kidney diseases. ${ }^{19,20}$ Nephrologists should be more interested in clinical data regarding the first month of life in the womb of their adult patients, being particularly focused on birth weight and on the weeks of 
gestation at birth, without forgetting data regarding maternal status during gestation and neonatal asphyxia. Neonatologists should be aware that any preterm or LBW infant should be considered as a subject with fewer glomeruli, probably predicted to develop renal disease later in life. The challenge for both is to develop an integrative approach, aimed at promoting an ongoing nephrogenesis after birth, at maintaining the self-renewal potential of renal SCs allowing them to originate new nephrons till the 38th postconceptional week, that represents the physiological stop for human nephrogenesis (-Fig. 1). The stimulation of endogenous renal SCs to produce new nephrons might represent a new relevant tool, able to improve nephron number in preterm and LBW newborns, allowing them to escape their susceptibility to undergo renal insufficiency later in life. The goal of our preventive regenerative renal approach, to be activated at birth, is to allow preterm and LBW infants to reach a sufficient number of functioning nephrons, protecting their nephrogenesis till the physiological end. With our approach, oligonephronic infants susceptible to develop renal failure in adulthood could be potentially transformed into normonephronic subjects, resistant to develop renal disease (-Fig. 2).

\section{Conflict of Interest}

None declared.

\section{References}

1 Faa G, Gerosa C, Fanni D, et al. Morphogenesis and molecular mechanisms involved in human kidney development. J Cell Physiol 2012;227(03):1257-1268

2 Faa G, Fanni D, Gerosa C, et al. The subcapsular blue strip: a new marker for evaluating the residual potential nephrogenesis in the newborn kidney. Mod Pathol 2013;26:387

3 Fanni D, Gerosa C, Van Eyken P, Gibo Y, Faa G. Development of the human kidney: immunohistochemical findings. In: Faa G, Fanos V, eds. Kidney Development in Renal Pathology. New York: Springer; 2014:29-41

4 Fanni D, Sanna A, Gerosa C, Puddu M, Faa G, Fanos V. Each niche has an actor: multiple stem cell niches in the preterm kidney. Ital J Pediatr 2015;41:78-83

5 Rodríguez MM, Gómez AH, Abitbol CL, Chandar JJ, Duara S, Zilleruelo GE. Histomorphometric analysis of postnatal glomer- ulogenesis in extremely preterm infants. Pediatr Dev Pathol 2004; 7(01):17-25

6 Puddu M, Fanos V, Podda F, Zaffanello M. The kidney from prenatal to adult life: perinatal programming and reduction of number of nephrons during development. Am J Nephrol 2009;30(02): $162-170$

7 Ingelfinger JR. Disparities in renal endowment: causes and consequences. Adv Chronic Kidney Dis 2008;15(02):107-114

8 Fanos V, Puddu M, Reali A, Atzei A, Zaffanello M. Perinatal nutrient restriction reduces nephron endowment increasing renal morbidity in adulthood: a review. Early Hum Dev 2010;86(Suppl 1):37-42

9 Faa G, Gerosa C, Fanni D, et al. Marked interindividual variability in renal maturation of preterm infants: lessons from autopsy. J Matern Fetal Neonatal Med 2010;23(Suppl 3):129-133

10 Bertram JF, Douglas-Denton RN, Diouf B, Hughson MD, Hoy WE. Human nephron number: implications for health and disease. Pediatr Nephrol 2011;26(09):1529-1533

11 Benigni A, Morigi M, Remuzzi G. Kidney regeneration. Lancet 2010;375(9722):1310-1317

12 Fanni D, Gerosa C, Nemolato S, et al. "Physiological" renal regenerating medicine in VLBW preterm infants: could a dream come true? J Matern Fetal Neonatal Med 2012;25(Suppl 3):41-48

13 Faa G, Gerosa C, Fanni D, et al. The role of immunohistochemistry in the study of the newborn kidney. J Matern Fetal Neonatal Med 2012;25(Suppl 4):135-138

14 Fanni D, Fanos V, Monga G, et al. MUC1 in mesenchymal-toepithelial transition during human nephrogenesis: changing the fate of renal progenitor/stem cells? J Matern Fetal Neonatal Med 2011;24(Suppl 2):63-66

15 Remuzzi A, Figliuzzi M, Bonandrini B, et al. Experimental evaluation of kidney regeneration by organ scaffold recellularization. Sci Rep 2017;7:43502-43505

16 Chevalier RL, Charlton JR. The human kidney at birth: structure and function in transition. In: Faa G, Fanos V, eds. Kidney Development in Renal Pathology. New York: Springer; 2014:49-58

17 Bertin M, Fanos V, Zanardo V. Malnutrition and renal function. In: Faa G, Fanos V, eds. Kidney Development in Renal Pathology. New York: Springer; 2014:95-102

18 Faa G, Fanos V. Renal endogenous stem cells: a new source for regenerative medicine in preterms? J Pediatr Neonat Individual Med 2015;4(02):e040224

19 Fanos V, Castagnola M, Faa G. Prolonging nephrogenesis in preterm infants: a new approach for prevention of kidney disease in adulthood? Iran J Kidney Dis 2015;9(03):180-185

20 Gerosa C, Fanni D, Faa A, et al. Low vascularization of the nephrogenic zone of the fetal kidney suggests a major role for hypoxia in human nephrogenesis. Int Urol Nephrol 2017;49(09): 1621-1625 